\title{
Clinical heterogeneity in autosomal dominant optic atrophy in two 3q28-qter linked central Illinois families
}

Angellee S. Chen, $M D^{l}$, Margaret J. Kovach, PhD ${ }^{l}$, Kristin Herman, $M D^{l}$, Arpenik Avakian, $M D^{3}$, William Frank, $M S^{2}$, Shawnia Forrester, $M S^{l}$, Jing-Ping Lin, $M D, P h D^{4}$, and Virginia Kimonis, $M D, M R C P^{l}$

\begin{abstract}
Purpose: To examine the clinical and genetic heterogeneity of autosomal dominant optic atrophy among two unrelated central Illinois families. Methods: Forty-three individuals from two pedigrees had complete eye examinations. Linkage analysis was performed with microsatellite markers from the region 3q28-29. Results: Visual acuity in 21 affected individuals ranged from 20/25 to 20/800. Vision loss was more severe in males than females $(P=0.02)$. Color vision testing revealed generalized dyschromatopsia. Both visual acuity and color vision deteriorated with age. Linkage was established to chromosome 3q28-29 (LOD $\max =4.68$ for D3S2305). Conclusion: Autosomal dominant optic atrophy linked to chromosome 3q28-29 shows intrafamilial phenotypic variation as well as sex-influenced severity in two Midwestern families. Genetics in Medicine, 2000:2(5):
\end{abstract}

283-289.

Key Words: optic atrophy, dominant, chromosome $3 q$, linkage, sex-influenced variation

Autosomal dominant optic atrophy, Kjer type (OPAl; MIM\# 16,5500 $)$ is an eye disorder first clinically described by Snell ${ }^{2}$ in 1897 and then again by Kjer in $1959 .{ }^{3}$ The disease has an incidence of approximately $1 / 50,000,{ }^{4}$ making it the most common form of inherited optic atrophy. Disease penetrance is nearly complete at $0.98 .{ }^{5} \mathrm{Kjer}$-type optic atrophy is suspected to be caused by diffuse, bilateral degeneration of the retinal ganglion cell layer of the eye with ascending demyelination and loss of nerve tissue on the temporal aspect of the optic nerves. ${ }^{3.6}$ The disease is clinically characterized by an insidious onset within the first decade of life of a symmetrical and progressive decrease in visual acuity, blue-yellow dyschromatopsia, and centrocecal scotomas of variable density. ${ }^{3}$ There is considerable heterogeneity in clinical symptoms of affected individuals, including reports of both inter- and intrafamilial phenotypic variability..$^{3,6-8}$ Reduction in visual acuity ranges from mild to severe visual impairment. In addition, optic disc involvement ranges from mild temporal pallor to complete atrophy of the optic nerve. ${ }^{9}$

Various studies have been conducted to define clinical characteristics of the disease and to identify the specific gene or

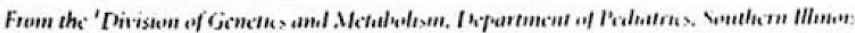

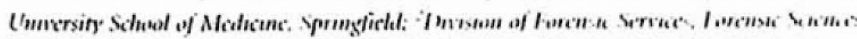

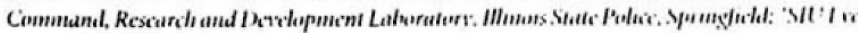

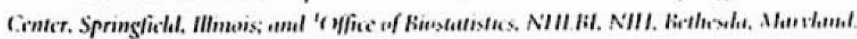

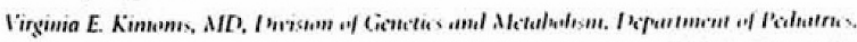

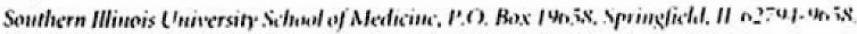
E-mail: vkimonis(arsiunnal.cilu.

Reccived: Juene 2n, $20 \mathrm{~km}$.

Acceptal: July 26. 2000 genes responsible for autosomal dominant optic atrophy. The OPAl gene locus was initially mapped to 3q28-qter by Eiberg et al. in 1994 by linkage analysis of three Danish families using dinucleotide repeat polymorphisms. ${ }^{10}$ Linkage to this region was also observed in four French pedigrees with autosomal dominant optic atrophy. ${ }^{11}$ The critical region was subsequently narrowed to the chromosome $3 q 28-29$ region by analysis of Cuban and British pedigrees. ${ }^{12.13}$ In 1997, the OPA1 gene locus was further refined the to a $1.4-\mathrm{cM}$ interval on chromosome 3q28-3q29 between markers D3S3669 and D3S3562 in a study of five Danish families with autosomal dominant optic atrophy. ${ }^{14} \mathrm{~A}$ founder effect of the dominant optic atrophy locus was demonstrated in a study of 38 pedigrees from the British Isles. ${ }^{15}$ This finding allowed for the application of linkage disequilibrium analysis to further refine the $O P A 1$ locus. These results suggest that OPA 1 lies within approximately 400 kb of marker D3S1523. Recently, analysis of a Western Maryland family of German descent has linked a second locus for dominant optic atrophy to chromosome 18q12.2-12.3, establishing the genetic heterogeneity of the disease. ${ }^{16}$

To date there has been only one previous linkage analysis of autosomal dominant optic atrophy in a United States family. In a study of a single, multigeneration Midwestern United States family, Brown et al. ${ }^{17}$ established linkage to the chromosome $3 q 28$ region. Our study presents both clinical and molecular analyses of two central Illinois families that demonstrate linkage to the chromosome 3q28-3q29 region. These data support previous findings that a single gene locus is responsible for the majority of dominant optic atrophy cases. 


\section{METHODS}

\section{Ascertainment of family members}

Informed consent was obtained from each participating subject from two central Illinois families with an autosomal dominant pattern of optic atrophy. Family A is a five-genera- tion family of Caucasian ancestry with 34 participants (19 males, 9 females, 6 spouses) ranging in age from 14 months to 65 years (Fig. la). Family B is a four-generation family of German origin with nine participants ( 2 males, 5 females, 2 spouses) (Fig. 1b). Ages ranged from 14 to 72 years.

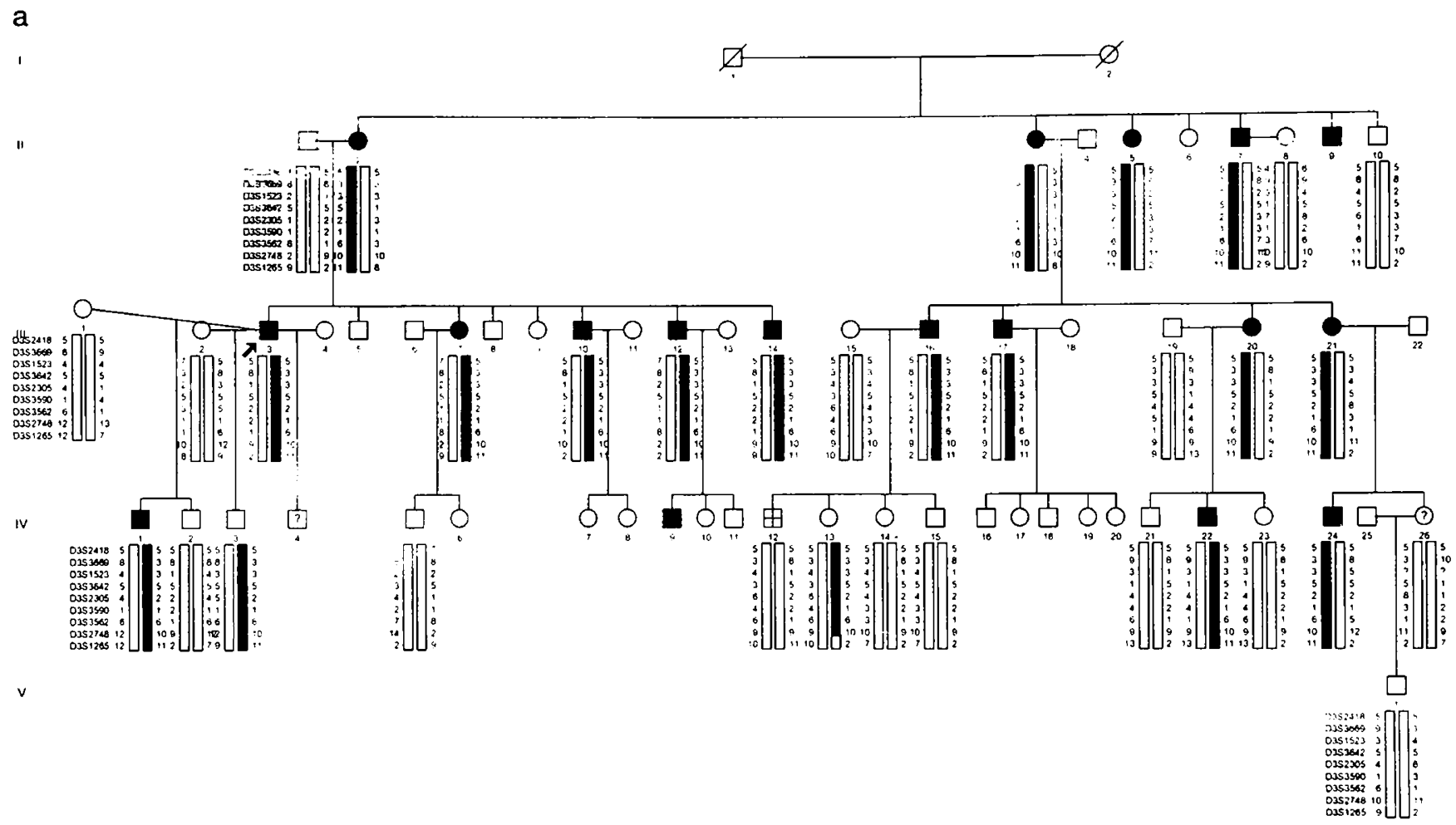

b

I

II

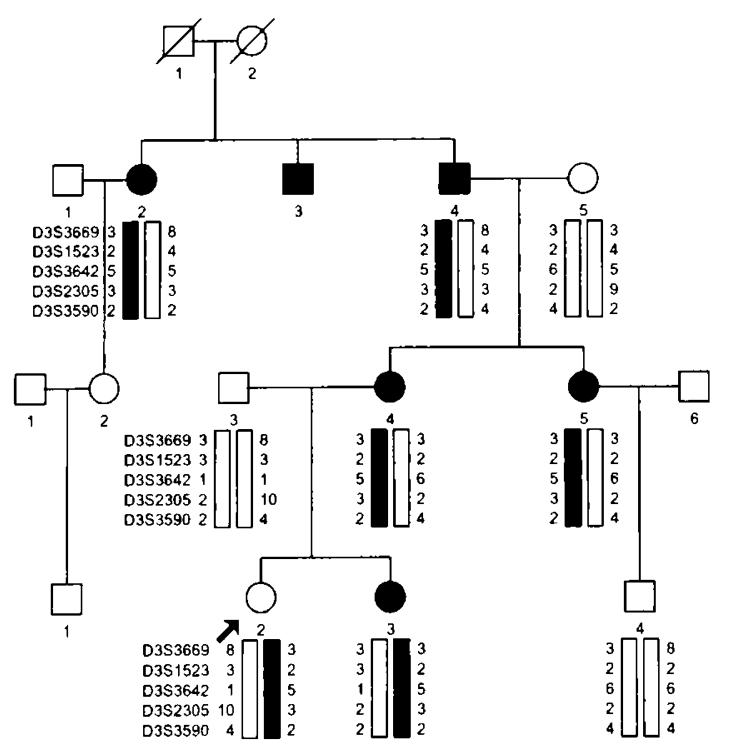

Fig. 1 Shown in a and b are pedigrees of two central Illinois families (A and B) with autosomal dominant optic atrophy showing haplotype analysis for microsatellite markers on chromosome 3q28-29. For each marker, alleles were numbered based on sizes reported in Genome Database (GDB). ${ }^{18}$ Novel alleles were assigned numbers following the last reported allele in GDB. Black bars represent the disease haplotype linked to autosomal dominant optic atrophy. Circles $=$ females, squares $=$ males, slashed symbols $=$ deceased, open symbols $=$ clinically unaffected, filled symbols = clinically affected, cross symbol = individual diagnosed with CHARGE syndrome, arrow indicates proband. In Family A, IV:13 appears clinically unaffected yet has inherited the disease haplotype. 


\section{Visual assessment}

Each subject was examined for visual acuity, color vision, and optic disc appearance. Visual acuity in each eye was evaluated based on best-corrected Snellen visual acuity. Color vision was assessed using Ishihara plates in all individuals and quantitated by the number of color plates accurately identified. The Farnsworth-Munsell 100-Hue test was additionally administered to seven individuals. Optic disc appearances for each subject were evaluated by direct funduscopic examination. Optic disc appearances were characterized as normal, or showing pallor, and/or excavation as determined by the examiner. Subjects were classified as clinically affected if they were an "at risk" individual greater than 6 years of age with reduced visual acuity $(<20 / 30)$, color vision deficit, and optic nerve pallor. Individuals were considered "at risk" if they were the offspring of people with definite optic atrophy and, therefore, were at $50 \%$ risk of inheriting the disease.

\section{Statistical analysis}

Visual acuity was evaluated by statistical methods including t-tests and regression analyses. Snellen acuity units were converted to the $\log$ of the minimum angle of resolution (logMAR units). The logMAR of 20/200 vision $=\log$ of $200 / 20$, or the log of $10=1$. Statistical significance was set at $5 \%$.

\section{DNA-marker analysis}

Blood samples, buccal scrapings, or both were collected from each subject. Genomic DNA was extracted from whole blood using a kit supplied by TEL-TEST, Inc. Buccal samples were processed using standard methods. Individuals were genotyped using nine polymorphic microsatellite markers on chromosome 3q28-29 overlapping the critical region of the OPA1 locus: D3S2418, D3S3669, D3S1523, D3S3642, D3S2305, D3S3590, D3S3562, D3S2748, and D3S1265. All forward primers were tagged with fluorescein dye using a $5^{\prime}$-oligolabeling kit (Amersham Pharmacia Biotech.) with the exception of D3S2305 and D3S1265. Markers D3S2305 and D3S1265 were obtained prelabeled from the manufacturer (Research Genetics, Inc.)

Polymerase chain reaction (PCR) amplification was carried out in a $25-\mu \mathrm{L}$ final reaction volume containing of 2-6 ng genomic DNA, 20 pmol each primer, $0.2 \mathrm{mM}$ each dNTP, $1 \mathrm{X}$ PCR buffer, $1.5 \mathrm{mM} \mathrm{MgCl}_{2}$, and $1 \mathrm{U}$ Taq DNA polymerase. Amplification was carried out for 35 cycles $\left(95^{\circ} \mathrm{C}\right.$ for 1 minute, $59-62^{\circ} \mathrm{C}$ for 1 minute, and at $72^{\circ} \mathrm{C}$ for 1 minute 30 seconds) followed by a final extension at $72^{\circ} \mathrm{C}$ for 7 minutes. Samples were analyzed on a $6 \% 6 \mathrm{M}$ urea denaturing polyacrylamide gel prepared in 1X TBE. Electrophoresis was carried out at $40 \mathrm{~W}$ for 1-3 hours. Fragments were visualized using an FM-BIO 100 fluorescent image-scanning unit (Hitachi).

\section{Linkage analysis}

Allele information was obtained from Genome Database. ${ }^{18}$ Marker-allele frequencies were estimated from the data by means of both observed and reconstructed genotypes of founders within the pedigrees. Two-point linkage analyses were carried out under an autosomal dominant model using the software LINKAGE. Multipoint linkage analyses were performed using LINKMAP. ${ }^{19}$ The genetic map derived by Votruba et al. ${ }^{15}$ was used to assign the order and relative genetic distances between the polymorphic markers in the multipoint analysis. Age-dependent penetrance was taken into account to allow for children who had not yet demonstrated any clinical evidence of optic atrophy. The five dependent risk classes identified were as follows: I, $0-10$ years old, 0.60 ; II, 11-20 years old, 0.80 ; III, $21-40$ years old, 0.90 ; IV, 40-60 years old, 0.95 ; and $\mathrm{V},>60$ years old, 0.99 .

\section{RESULTS}

\section{Clinical evaluation}

Two central Illinois families, one of mixed Caucasian descent and the other of German ancestry, were used in a clinical and molecular study to evaluate the phenotypic and genetic heterogeneity of dominant optic atrophy. Table 1 summarizes the clinical findings of both families. In Family A, 17 individuals ( $11 \mathrm{M}, 6 \mathrm{~F})$ subjected to visual assessment had clinical findings consistent with optic atrophy with visual acuity among family members ranging from 20/25 to 20/800 with poor color vision in older individuals. The proband, individual III:3, was diagnosed with optic atrophy at age 17 , although he admitted having visual difficulties from the age of 7 years. He attended a school for the blind and visually impaired from an early age. His recent visual acuity was 20/400 OD and 20/400+ OS, and his color vision was also poor (Ishihara 1/14). He had markedly pale optic discs with large optic cups. Brain and optic nerve magnetic resonance imaging was normal. His son (IV:1) age 6 years, was clinically diagnosed with optic atrophy after experiencing difficulty with his vision in the classroom setting. His visual acuity was $20 / 60 \mathrm{OU}$; however, his color vision was normal. The youngest individual in Family A diagnosed with optic atrophy was IV:22, a 51/2 year old male who had failed a vision screening test administered at his school. On subsequent ophthalmological evaluation, his visual acuity was $20 / 60 \mathrm{OU}$ and optic nerve pallor was apparent. He scored 11/14 on Ishihara color vision testing.

The clinical variability and incomplete penetrance of dominant optic atrophy makes diagnosis of the disorder difficult to establish in some individuals. For example, individual II:3, a 61 year old female and obligate carrier of the OPAl gene, had normal visual acuity and moderately preserved color vision. In contrast, two individuals in Family A had an early onset of symptoms, presenting at ages 6 and 7 years. Visual impairment in individual IV:12 was difficult to interpret, given his existing diagnosis of CHARGE syndrome, a complex disorder of unknown etiology characterized by Coloboma of the eyes, Heart defects, Atresia of the choanae, Retarded growth and development, Genital hypoplasia, and Ear anomalies or hearing loss. This patient exhibited coloboma of his right optic disc, patent ductus arteriosis, a visual acuity of 20/40, and normal color vision. 
Table 1

Clinical characteristics of affected individuals in two central Illinois pedigrees with autosomal dominant optic atrophy

\begin{tabular}{|c|c|c|c|c|c|}
\hline Pedigree No. & Age & Sex & Visual acuity & Color vision & Ophthalmoscopy findings \\
\hline \multicolumn{6}{|l|}{ Family A } \\
\hline II: 2 & 60 & $\mathrm{~F}$ & $20 / 200$ OD; $20 / 100$ OS & $0 / 14 \mathrm{OU}$ & Pallor with sloping excavation \\
\hline II:3 & 61 & $\mathrm{~F}$ & $20 / 25 \mathrm{OD} ; 20 / 30 \mathrm{OS}$ & $7 / 13 \mathrm{OD} ; 10 / 13 \mathrm{OS}$ & Pallor with excavation \\
\hline II:5 & 57 & $\mathrm{~F}$ & $20 / 100$ OD; $20 / 70$ OS; $20 / 40$ OU & $0 / 14 \mathrm{OU}$ & Optic pallor \\
\hline II:7 & 60 & M & $20 / 800 \mathrm{OU}$ & $0 / 14 \mathrm{OU}$ & Optic pallor \\
\hline III: 3 & 27 & $\mathrm{M}$ & $20 / 400 \mathrm{OU}$ & $1 / 14 \mathrm{OU}$ & Pallor with large optic discs \\
\hline III:7 & 37 & $\mathrm{~F}$ & $20 / 100 \mathrm{OU}$ & $0 / 14 \mathrm{OU}$ & Pallor with mild excavation \\
\hline III 10 & 35 & M & $20 / 200 \mathrm{OU}$ & $0 / 14 \mathrm{OU}$ & Optic atrophy \\
\hline III: 12 & 33 & M & $20 / 200 \mathrm{OU}$ & $0 / 14 \mathrm{OU}$ & Optic atrophy \\
\hline III 14 & 32 & M & $20 / 200$ OD; $20 / 100$ OS & $0 / 14 \mathrm{OU}$ & $\begin{array}{l}\text { Pallor with marked } \\
\text { excavation }\end{array}$ \\
\hline III:16 & 40 & M & $20 / 400 \mathrm{OU}$ & $0 / 14 \mathrm{OU}$ & Disc pallor \\
\hline III: 17 & 37 & M & $20 / 200 \mathrm{OU}$ & $0 / 14 \mathrm{OU}$ & Disc pallor \\
\hline III: 20 & 30 & $\mathrm{~F}$ & $20 / 80$ OU & $1 / 14 \mathrm{OU}$ & Pallor with excavation \\
\hline III:21 & 43 & $\mathrm{~F}$ & $20 / 400$ OU & $0 / 14 \mathrm{OU}$ & Disc pallor \\
\hline IV:1 & 6 & M & $20 / 60 \mathrm{OU}$ & $14 / 14$ OD; $13 / 14$ OS & Marked temporal pallor \\
\hline IV:13 & 19 & $\mathrm{~F}$ & $20 / 40$ OD $; 20 / 20$ OS & $12 / 12$ OD; $14 / 14$ OS & Normal \\
\hline IV:22 & 5.5 & M & $20 / 60 \mathrm{OU}$ & $11 / 14 \mathrm{OU}$ & Disc pallor \\
\hline IV:24 & 26 & M & $20 / 200 \mathrm{OU}$ & $10 / 12 \mathrm{OU}$ & Disc pallor \\
\hline \multicolumn{6}{|l|}{ Family B } \\
\hline II:2 & 70 & $M$ & $20 / 200$ at $35^{\prime \prime} \mathrm{OU}$ & $0 / 14 \mathrm{OU}$ & Advanced optic atrophy \\
\hline II:4 & 72 & M & $20 / 400 \mathrm{OU}$ & $0 / 14 \mathrm{OU}$ & Advanced optic atrophy \\
\hline III:4 & 50 & $\mathrm{~F}$ & $20 / 200 \mathrm{OU}$ & $0 / 14$ OU & Advanced optic atrophy \\
\hline III:5 & 35 & $\mathrm{~F}$ & $20 / 200 \mathrm{OU}$ & $13 / 14 \mathrm{OU}$ & Advanced optic atrophy \\
\hline IV:2 & 13 & $\mathrm{~F}$ & $20 / 20 \mathrm{OU}$ & $14 / 14 \mathrm{OU}$ & Normal discs \\
\hline IV:3 & 14 & $\mathrm{~F}$ & $20 / 50 \mathrm{OU}$ & $14 / 14 \mathrm{OU}$ & Optic atrophy \\
\hline
\end{tabular}

$\mathrm{OD}$, right eye; OS, left eye; $\mathrm{OU}$, both eyes.

Visual acuity was assessed by Snellen analysis and color vision was assessed using Ishihara charts.

Ishihara testing revealed that all individuals age $>27$ years (except II:3, age 61 years) revealed poor color vision $(\leq 1 / 14)$. The Farnsworth-Munsell 100-Hue test, administered to six individuals in Family A, demonstrated generalized dyschromatopsia.

The proband for Family B (IV:2) is a 13 year old girl with learning disabilities, dysmorphic features, subglottic hemangiomas, immunoglobulin deficiencies, and failure to thrive. Dysmorphological evaluation failed to establish a diagnosis, and karyotype and basic metabolic studies on her were normal. Her visual acuity was $20 / 20$, and funduscopic examination was normal. Although she had no visual deficits on evaluation, her family history determined that she was at risk of inheriting autosomal dominant optic atrophy. In total, seven individuals were examined in Family B, five of which were identified as affected upon visual examination $(2 \mathrm{M}, 3 \mathrm{~F})$. Visual acuity of affected individuals ranged from 20/50 (IV:3) to 20/400 (II:4), and optic disc atrophy was moderate to advanced. Her sister, age 14, was found to have poor visual acuity and color vision, as well as features of optic disc atrophy.

Interestingly, vision loss among affected males was observed to be more severe than among affected females. The Student's T-test established that there was no significant difference in ages between the genders of both study groups; however, the $\log$ visual acuity between genders was statistically significant $(P=0.02)$. Figure $2 \mathrm{~A}$ depicts a scatterplot of age versus visual acuity comparing affected males to affected females. There were no statistical differences in the slopes of the linear regressions between the genders when predicting visual acuity from age, although there was still a difference in the mean visual acuity $(P<0.05)$. In addition, Figures $2 \mathrm{~B}$ and $2 \mathrm{C}$ present the results, including $95 \%$ prediction intervals, when a quadratic component was added to the model. This analysis increases the $\mathrm{R}^{2}$ value from 0.09 for females and 0.49 for males to approxi- 

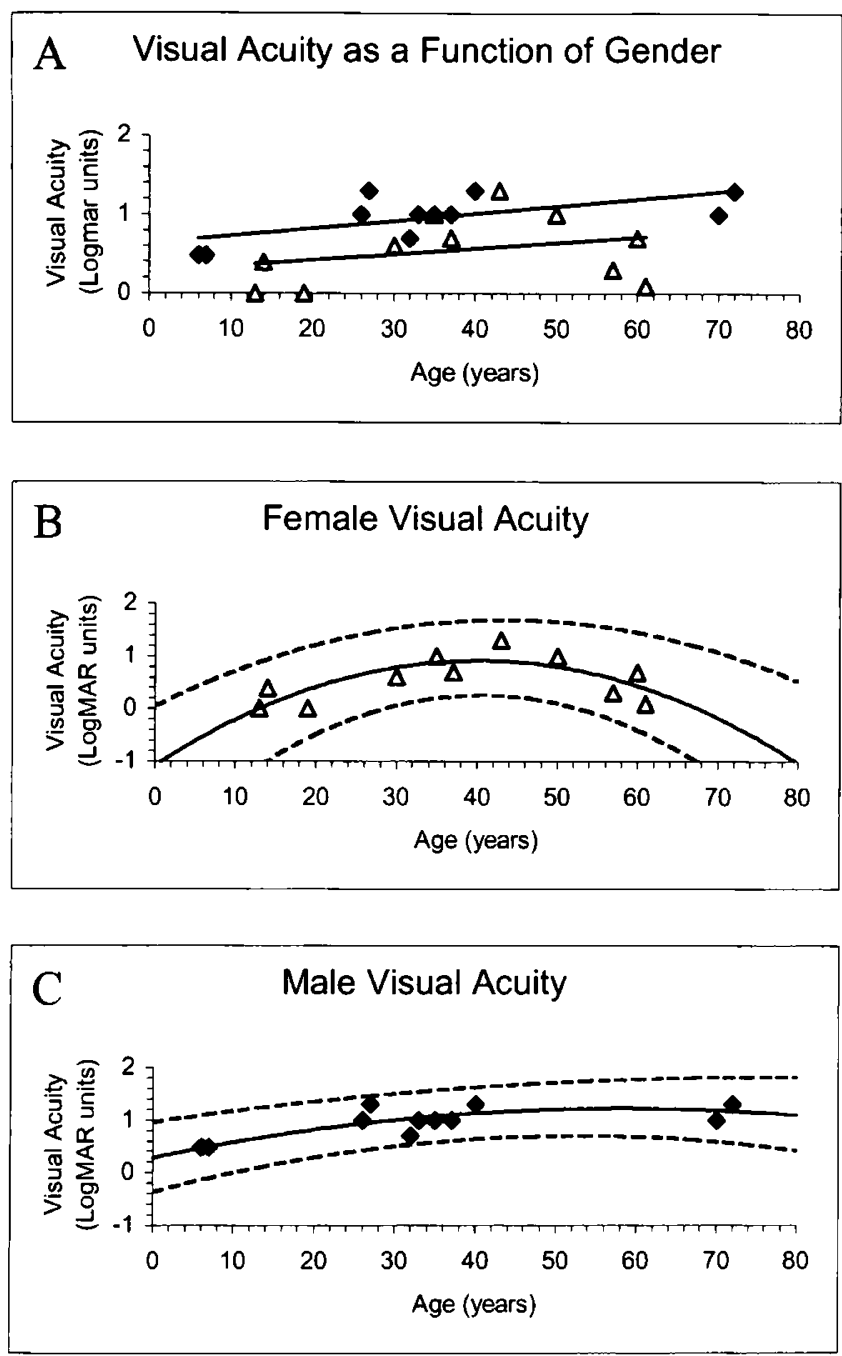

Fig. 2 Scatterplot of age versus visual acuity comparing affected males and affected females. Panel A depicts linear regression of visual acuity plotted as a function of age for affected fermales versus affected males. Panels $B$ and $C$ present visual acuity data with a quadratic component added to the model. The $95 \%$ prediction interval for each data set is represented by dashed lines. Triangles denote female data; diamonds denote male data.

mately 0.61 for both, thereby increasing the predictive ability of the model and also indicating a significant difference between genders $(P<0.001)$.

\section{Genetic linkage and haplotype analysis}

Genotyping of both families with microsatellite markers established linkage to the OPA1 locus on chromosome $3 \mathrm{q} 28$ q29. Table 2 presents the LOD scores derived from two-point linkage analysis of Families A and B. A maximum combined LOD score of 4.68 at $\theta=0.0$ was obtained with marker D3S2305. Multipoint analysis of both families generated a maximum LOD score of 5.82 positioned within the D3S2305D3S3590 interval (Fig. 3). The disease haplotype was reconstructed via visual inspection, assigning the most likely linkage phase by minimizing the number of recombinations within a pedigree (Figs. 1a and $1 \mathrm{~b}$ ). In Family A, a recombination between markers D3S1265 and D3S2748 (IV:13, Family A) ex-
Table 2

Two-point LOD scores using markers from chromosome $3 q$

\begin{tabular}{lcccccc}
\hline Marker & 0.00 & 0.10 & 0.20 & 0.30 & 0.40 & 0.50 \\
\hline Family A & & & & & & \\
D3S2418 & -0.27 & 0.23 & 0.26 & 0.18 & 0.08 & 0.00 \\
D3S3669 & 1.84 & 1.57 & 1.23 & 0.84 & 0.41 & 0.00 \\
D3S1523 & 2.30 & 1.94 & 1.50 & 1.01 & 0.49 & 0.00 \\
D3S3642 & 2.38 & 1.98 & 1.53 & 1.01 & 0.42 & 0.00 \\
D3S2305 & 4.40 & 3.64 & 2.78 & 1.82 & 0.77 & 0.00 \\
D3S3590 & 1.11 & 1.04 & 0.91 & 0.71 & 0.41 & 0.00 \\
D3S3562 & 3.18 & 2.76 & 2.21 & 1.52 & 0.71 & 0.00 \\
D3S2748 & 1.35 & 1.17 & 0.98 & 0.74 & 0.41 & 0.00 \\
D3S1265 & 4.34 & 3.69 & 2.90 & 1.99 & 0.94 & 0.00 \\
Family B & & & & & & 0.00 \\
D3S3669 & 0.38 & 0.27 & 0.17 & 0.08 & 0.02 & 0.00 \\
D3S1523 & 0.50 & 0.35 & 0.21 & 0.10 & 0.03 & 0.00 \\
D3S3642 & 0.10 & 0.15 & 0.15 & 0.12 & 0.07 & 0.00 \\
D3S2305 & 0.28 & 0.27 & 0.23 & 0.16 & 0.08 & 0.00 \\
D3S3590 & 0.41 & 0.21 & 0.07 & 0.01 & -0.00 & 0.00 \\
\hline
\end{tabular}

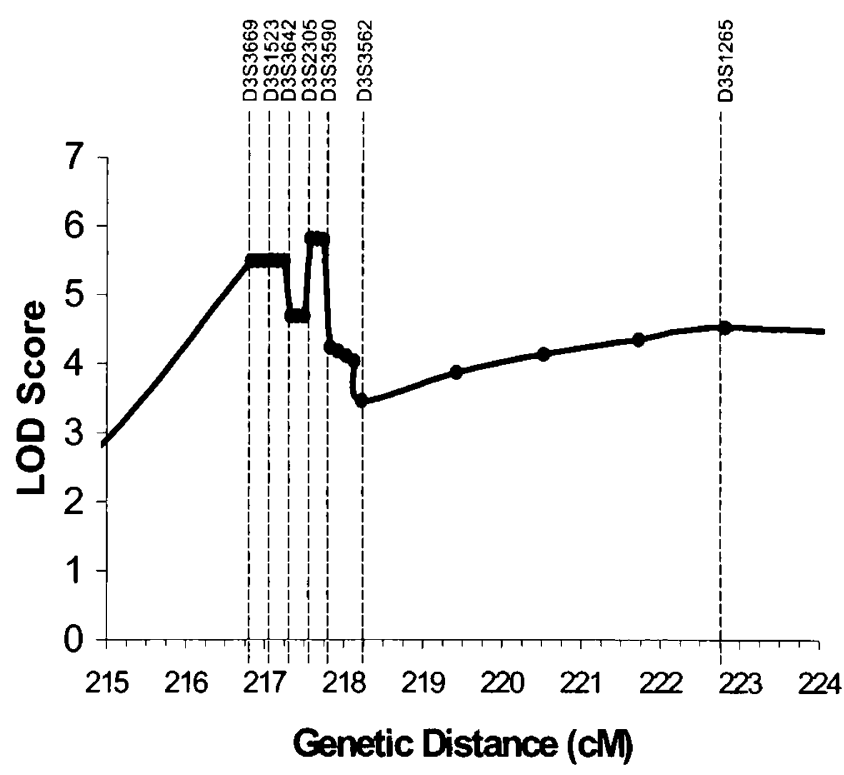

Fig. 3 Multipoint linkage analysis of the OPAl region using microsatellite markers around the OPAl locus. Approximate marker positions are based on the Marshfield genetic map and are represented by dashed lines.

cluded the region telomeric of D3S2748. Individual IV:13, a female 19 years old, had no evidence of optic atrophy. Her visual acuity was 20/40 OD and 20/20 OS and her color vision was normal. She is presumed to be an asymptomatic carrier of disease, because she has the disease haplotype encompassing the critical region as defined in previous reports. Individual IV:12, who had CHARGE syndrome and visual impairment, 
did not inherit the disease haplotype from his affected father. In Family B, a disease haplotype was assigned; however, there were no informative recombinations. Although the proband in Family B had clearly inherited her mother's disease haplotype, she does not exhibit any signs of optic atrophy. The clinical status of all other individuals coincided with the results of the haplotype analysis. Figure 4 summarizes conclusions derived from haplotype analysis and previous reports defining the $O P A l$ genetic locus.

It was noted that the haplotype designation for markers within the critical region of the OPAI locus differs between Family A and B. Since the distance spanning the markers examined is relatively small $(1.4 \mathrm{cM})$, it is likely that genetic mutations resulting in disease manifestations in these two families arose independently of each other, and the same founder is not responsible for dominant optic atrophy in these two central Illinois pedigrees.

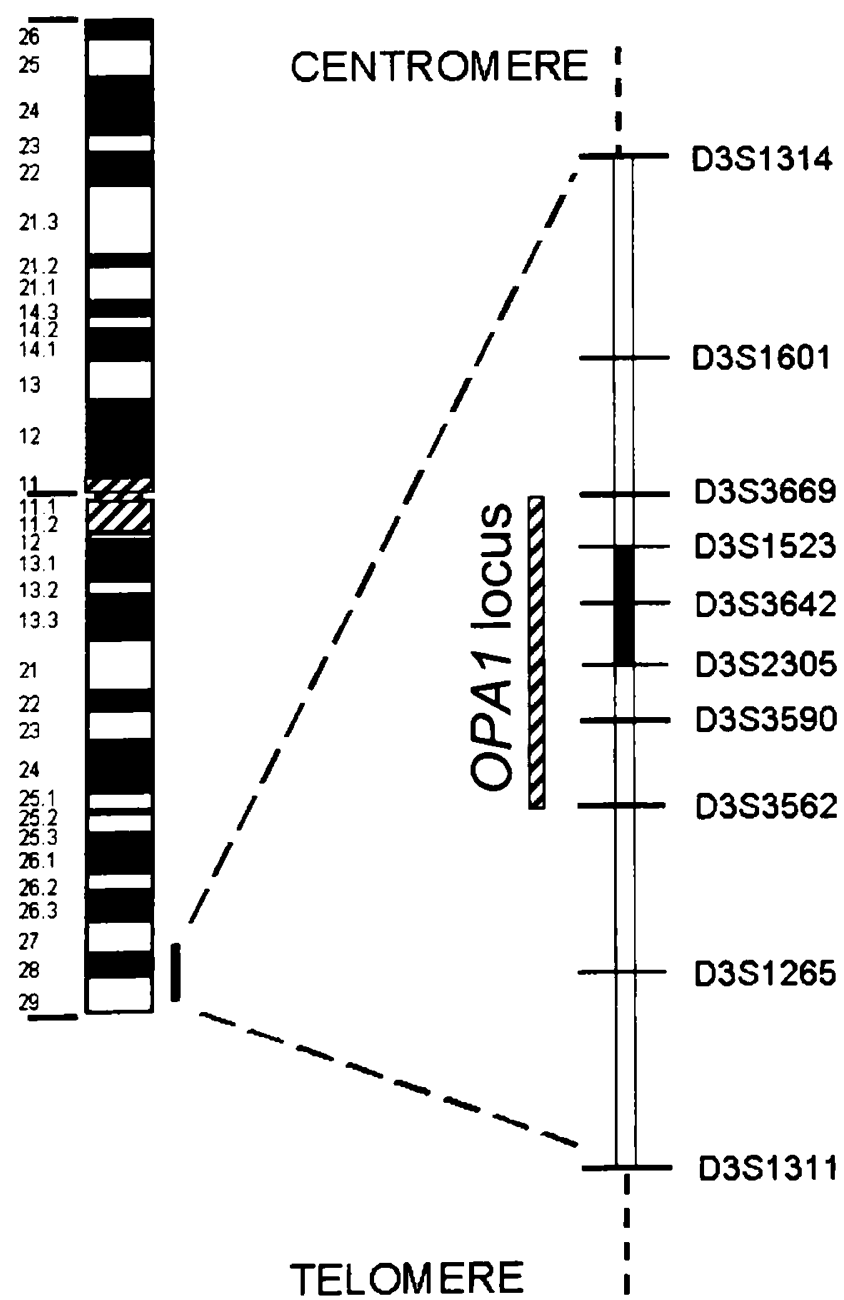

Fig. 4 Schematic diagram of chromosome 3 depicting markers spanning the critical region of the OPAI disease locus. The OPAI locus defined by haplotype analysis is represented by a hatched bar. ${ }^{14}$ The critical region defined by linkage disequilibrium analysis $^{15}$ is denoted by a solid black bar on the expanded skeleton of chromosome 3q28-q29.

\section{DISCUSSION}

This cross-section study in two families with autosomal dominant optic atrophy show intrafamilial clinical variability and a more severe phenotype among older individuals for both color and visual acuity. Clinical features in affected individuals ranged from near normal visual acuity and relatively preserved color vision (II:3, Family A) to severe loss of vision in a 60 year old male with who was diagnosed with optic atrophy in his $20 \mathrm{~s}$, and suffered from diabetes and strokes (II:7, Family A) (Table 1). Males were found to be more severely affected than females in our families, as indicated by the log visual acuity between the genders. A more striking sex-influenced difference in disease severity was previously observed by Gorgone et al..$^{20}$ In their study of 20 affected members of a single, three-generation family with dominant optic atrophy, the authors observed a bimodal distribution of disease severity. All four of the severely affected individuals were males, whereas the remaining 16 individuals ( $7 \mathrm{M}, 9 \mathrm{~F})$ were asymptomatic with mild optic atrophy. No clear genetic or environmental factor has been identified that would explain this sex difference, but it could be due to the influence of epigenetic factors.

In addition to the primary disease locus on chromosome $3 q 28-q 29$, genetic heterogeneity of autosomal dominant optic atrophy recently has been demonstrated with linkage to chromosome 18.16 Molecular analysis of the two families in this study established linkage to chromosome 3q28-qter. Informative recombinations were not observed upon haplotype analysis of either pedigree examined in this study, thereby precluding further refinement of the OPA gene locus. Multipoint analysis, however, determined that the gene most likely resides in the interval flanked by markers D3S2305 and D3S3590, a finding consistent with other reports.

Despite refinement of the OPAl locus to within $400 \mathrm{~kb}$ of D $9 S 1523,7$ the gene responsible for this disorder remains unknown. Two candidate genes have been excluded, however, through mutational and haplotype analysis. The DAGK3 gene (diacylglycerol kinase 3), predominantly expressed in the human retina, ${ }^{21}$ has been mapped by in situ hybridization to human chromosome 3q27-q28.22 In Drosophila melanogaster, mutations in an eye-specific diacylglycerol kinase have been shown to be associated with retinal degeneration. ${ }^{23}$ Mutational analysis of DAGK3 in 19 unrelated German OPA1 patients, however, did not reveal any disease-associated mutations..$^{22}$ In addition, the $H R Y$ gene, which encodes a basic helix-loop-helix protein, also maps to chromosome 3q28-q29; however, Votruba et al. ${ }^{24}$ described recombinations between HRY and $O P A 1$, thereby excluding it as a candidate gene.

Identification of genetic mutations and other genetic and environmental factors involved in dominant optic atrophy, will help to explain the clinical and gender variability observed in affected individuals through phenotype:genotype correlation. This information will also contribute to our understanding of the pathogenesis of autosomal dominant optic atrophy, possibly permitting development of future effective therapies for the disease. 


\section{Acknowledgments}

This project was funded by a grant from the Central Research Committee at the Southern Illinois University School of Medicine, Springfield, IL. We thank both families for their cooperation and participation in this study; Dr. Steven Verhulst for the statistical analysis; Dr. Lance Steahley, Dr. Robert Enzenauer, and Dr. Theobald for their clinical evaluations of the patients; and Dr. Votruba and the Illinois State Police Laboratory for their contributions.

\section{References}

1. MIM (Online Mendelian inheritance in man) Baltimore: Johns Hopkins University, Center for Medical Genetics, 2000. http://www3.ncbi.nlm.nih.gov/omim/(March).

2. Snell S. Diseases of the optic nerve. I. Hereditary or congenital optic atrophy and allied cases. Trans Ophthal Soc UK 1897;17:66-81.

3. Kjer P. Infantile optic atrophy with dominant mode of inheritance: a clinical and genetic study of 19 Danish families. Acta Ophthalmol Scand 1959;(Suppl 54):1-1 46

4. Lyle WM. Genetic risks. Waterloo, Ontario: University of Waterloo Press, 1990.

5. Kivlin JD, Lovrien EW, Bishop T, Maumenee IH. Linkage analysis in dominant optic atrophy. Am J Hum Genet 1983;35:1190-1195.

6. Johnston PB, Gaster RN, Smith VC, Ramesh CT. A clinicopathologic study of autosomal dominant optic atrophy. Am J Ophthalmol 1979;88:868-875.

7. Votruba M, Fitzke FW, Holder GE, Carter A, Bhattacharya SS, Moore AT. Clinical features in affected individuals from 21 pedigrees with dominant optic atrophy. Arch Ophthalmol 1998;116:351-358.

8. Elliott D, Traboulsi EI, Maumenee IH. Visual prognosis in Kjer type autosomal dominant optic atrophy. Am J Ophthalmol 1993:115:360-367.

9. Johnston RL, Seller MJ, Behnam IT, Burdon MA. Spalton DJ. Dominant optic atrophy: refining the clinical diagnostic criteria in light of genetic linkage studies. Ophthalmology 1999;106:123-128.

10. Eiberg H, Kjer B, Kjer P, Rosenberg T. Dominant optic atrophy (OPA1) mapped to chromosome 3q region. I. Linkage analysis. Hum Mol Genet 1994;3:977-980.

11. Bonneau D, Souied E, Gerber S, Rozet JM, D'Haens E, Journel H, Plessis G, Weissenbach I, Munnich A, Kaplan J. No evidence of genetic heterogeneity in dominant optic atrophy. J Med Genet 1995;32:951-953.

12. Lunkes A, Hartung U, Magarino C, Rodriguez M, Palmero A, Rodriguez L, Heredero L, Weissenbach J, Weber J, Auburger G. Refinement of the OPAl gene locus on chromosome $3 \mathrm{q} 28-\mathrm{q} 29$ to a region of $2-8 \mathrm{cM}$, in one Cuban pedigree with autosomal dominant optic atrophy type Kjer. Am J Hum Genet 1995;57:968-970.

13. Votruba M, Moore AT, Bhattacharya SS. Genetic refinement of dominant optic atrophy (OPA1) locus to within a $2 \mathrm{cM}$ interval of chromosome 3q. J Med Genet 1997;34:117-121.

14. Jonasdottir A, Eiberg H, Kjer B, Kjer P, Rosenberg T. Refinement of the dominant optic atrophy locus (OPA1) to a l.4-cM interval on chromosome $3 \mathrm{q} 28-3 \mathrm{q} 29$, within a 3-Mb YAC contig. Hum Genet 1997;99:115-120.

15. Votruba M, Moore AT, Bhattacharya SS. Demonstration of a founder effect and fine mapping of dominant optic atrophy locus on $3 \mathrm{q} 28$-qter by linkage disequilibrium method: a study of 38 British Isles pedigrees. Hum Genet 1998;102:79-86

16. Kerrison JB, Arnould VJ, Ferraz Sallum JM, Vagefi MR, Barmada MM, Li Y, Zhu D Maumenee IH. Genetic heterogeneity of dominant optic atrophy, Kjer type: identification of a second locus on chromosome 18q12.2-12.3. Arch Ophthalmol 1999. 117:805-810.

17. Brown J, Fingert JH, Taylor CM, Lake M, Sheffield VC, Stone EM. Clinical and genetic analysis of a family affected with dominant optic atrophy (OPA 1$)$. Arch Ophthalmol 1997;115:95-99.

18. http.//www.gdb.org.

19. O'Connell JR, Weeks DE. The VITESSE algorithm for rapid exact multilocus linkage analysis via genotype set-recoding and fuzzy inheritance. Nat Genet 1995:11:402408.

20. Gorgone G, Li Volte S, Cavallaro N, Conti L, Profeta GM, Mollica F. Familial optic atrophy with sex-influenced severity: a new variety of autosomal-dominant optic atrophy. Ophthalmologica 1986;192:28-33.

21. Kai M, Sakane F, Imai S, Wada I, Kanoh H. Molecular cloning of a diacylglycerol kinase isozyme predominantly expressed in human retina with a truncated and inactive enzyme expression in most other human cells. / Biol Chem 1994;269:1849218498.

22. Stohr H, Klein I, Gehrig A, Koehler MR, Jurklies B, Kellner U, Leo-Kottler B, Schmid $\mathrm{M}$, Weber $\mathrm{BH}$. Mapping and genomic characterization of the gene encoding diacylglycerol kinase gamma ( $\left.D A G K^{\prime} 3\right)$ : assessment of its role in dominant optic atrophy (OPA1). Hum Genet 1999;104:99-105.

23. Masai I, Okazaki A. Hosoya T, Hotta Y. Drosophila retinal degeneration A gene encodes an eye-specific diacylglycerol kinase with cysteine-rich zinc-finger motif and ankyrin repeats. Proc Nat Acad Sci USA 1993;90:11157-11161.

24. Votruba M, Payne A, Moore AT, Bhattacharya SS. Dominant optic atrophy: exclusion and fine genetic mapping of the candidate gene, HRY. Mamm Genome 1998;9: $784-787$. 